\title{
ASSISTÊNCIA FARMACÊUTICA EM DROGARIAS: IMPORTÂNCIA, DESAFIOS E IMPEDIMENTOS
}

\author{
Aurileane Carneiro da Silva ${ }^{1}$ \\ Maria Betânia Barbosa de Souza ${ }^{2}$ \\ Glícia Maria de Oliveira ${ }^{3}$ \\ Jaiurte Gomes Martins da Silva ${ }^{4}$ \\ Girliane Regina da Silva ${ }^{5}$
}

RESUMO: Com a expansão $d a$ indústria farmacêutica, o profissional farmacêutico sofreu uma descaracterização de suas funções, foi levado a se distanciar desse contato direto com a sociedade. A dispensação de medicamentos se tornou um ato mecânico, desprovido dos cuidados necessários para a assistência à saúde. A execução dessas práticas nas drogarias pode influenciar positivamente em resultados terapêuticos satisfatórios. Trata-se de uma revisão de literatura, que permite a combinação de dados da literatura empírica e teórica, realizada no período entre fevereiro e outubro de 202I, no qual foram adotados materiais devidamente publicados. O papel chave do Farmacêutico é estender o caráter de beneficiário da Atenção Farmacêutica ao público, em seu conjunto, e reconhecer, deste modo, o farmacêutico como dispensador da atenção sanitária que pode participar, ativamente, na prevenção das doenças e da promoção da saúde, junto com outros membros da equipe sanitária. De modo que, para se dispensar medicamentos corretamente, é importante manter um diálogo entre o paciente e o profissional farmacêutico. O reconhecimento da contribuição da atuação deste profissional para a obtenção de melhores resultados em saúde respalda a importância do farmacêutico nas drogarias e farmácias.

Palavras-chave: Farmacêutico. Drogarias. Dispensação de Medicamentos.

\footnotetext{
${ }^{1}$ Discentes do curso de Bacharelado em Farmácia da Faculdade Santíssima Trindade - FAST.

${ }^{2}$ Discentes do curso de Bacharelado em Farmácia da Faculdade Santíssima Trindade - FAST.

${ }^{3}$ Enfermeira, Mestre pela Universidade Federal de Pernambuco - UFPE; Docente da Faculdade Santíssima Trindade - FAST

${ }^{4}$ Biólogo, Mestre pela Universidade Federal Rural de Pernambuco - UFRPE; Docente da Faculdade Santíssima Trindade - FAST.

${ }^{5}$ Farmacêutica, Doutora pela Universidade Federal Rural de Pernambuco - UFRPE; Docente da Faculdade Santíssima Trindade - FAST.
} 
ABSTRACT: With the expansion of the pharmaceutical industry, the pharmacist suffered a mischaracterization of their functions, they were led to distance themselves from this direct contact with society. The dispensing of medication has become a mechanical act, devoid of medical care for health care. An implementation of these practices in drugstores can positively lead to satisfactory therapeutic results. This is a literature review, which allows the combination of empirical and theoretical literature data, carried out in the period between February and October 2021, in which duly published materials were adopted. The key role of the Pharmacist is to extend the beneficiary character of Pharmaceutical Care to the public, as a whole, and thus recognize the pharmacist as a health care provider who can actively participate in disease prevention and health promotion, together with other members of the health team. So, in order to dispense medication correctly, it is important to maintain a dialogue between the patient and the pharmacist. The recognition of the contribution of this professional's performance to obtain better health outcomes supports the importance of the pharmacist in drugstores and pharmacies.

Keywords: Pharmacist. Drugstores. Drug Dispensing.

\section{INTRODUÇÃO}

Nos primórdios da profissão farmacêutica, havia um contato direto desse profissional com a sociedade, as formulações eram feitas de forma individualizadas para cada paciente de acordo com a suas patologias, e as orientações sobre os medicamentos, assim como, outros produtos com fins terapêuticos, que o mesmo produzia era uma prática constante e natural do ofício. As mudanças ocorridas ao longo do tempo no campo da farmácia após a industrialização do setor de medicamentos, proporciou um distanciamento do farmacêutico das suas atividades tradicionais: a manipulação de medicamentos e o atendimento ao paciente (ZUBIOLI, 2004, ALENCAR et al, 20Ir; OLIVEIRA et al, 2017).

Também com a expansão da indústria farmacêutica, o profissional farmacêutico sofreu uma descaracterização de suas funções, foi levado a se distanciar desse contato direto com a sociedade, concentrando-se nas indústrias principalmente, além da dificuldade em empreender e dado ao aumento da concorrência causado pelo crescimento de Farmácias de Rede, houve uma descaracterização das funções do farmacêutico junto à sociedade. A dispensação 

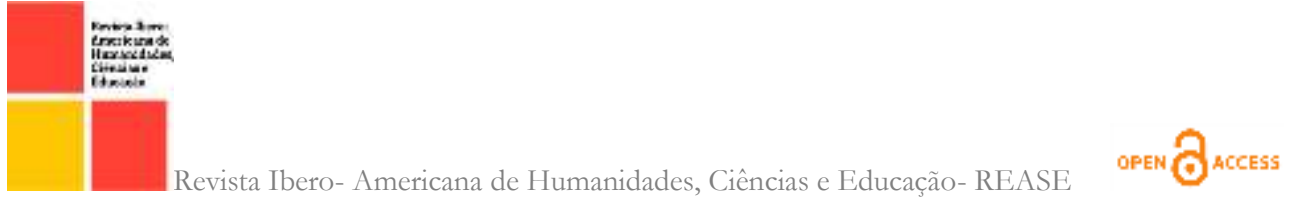

de medicamentos se tornou um ato mecânico, desprovido dos cuidados necessários para a assistência à saúde, havendo, contudo, uma tendência de incorporação de novas práticas como: ouvir as necessidades do paciente bem como instruir ao uso racional de medicamentos, necessitando ser resgatado o papel principal do farmacêutico como dispensador para um maior ganho social (ALENCAR et al, 2oIr; OLIVEIRA et al, 2017).

O cenário farmacêutico no Brasil tem passado por mudanças importantes nos últimos anos, todas elas resgatando o valor e o papel do farmacêutico na sociedade. A prática da atenção farmacêutica, assim denominada, é um novo modelo e uma das funções primordiais do profissional farmacêutico, que é a função assistencial, a qual, trata-se da interação direta do farmacêutico com o usuário/cliente, visando uma farmacoterapia racional e funcional, bem como, a obtenção de resultados definidos e mensuráveis, voltados para uma melhoria na qualidade de vida (STEFANO, ARRUDA, 2018; RODRIGUES, 2019; SANTOS et al, 2020).

Visto que, o uso de medicamentos sem orientação técnica pode ocasionar danos à saúde, tais como: efeitos indesejáveis, agravamento e mascaramento de doenças, interações, dependência, reações alérgicas, resistência bacteriana e até mesmo levar a óbito (GASPAR, MACHADO 2015; PEDRO et al, 2020).

A dispensação de medicamentos e a qualidade dessa prática podem ser consideradas abaixo do padrão, uma vez que os farmacêuticos frequentemente estão ausentes das drogarias ou não executam corretamente tais atividades de sua responsabilidade (OLIVEIRA et al, 2017; PEDRO et al, 2020). O presente trabalho tem como objetivo, identificar a importância e as dificuldades da assistência farmacêutica, na dispensação de medicamentos em drogarias, sob supervisão do profissional farmacêutico.

\section{METODOLOGIA}

Trata-se de uma revisão de literatura, do tipo narrativa, onde permite a combinação de dados da literatura empírica e teórica que podem ser 

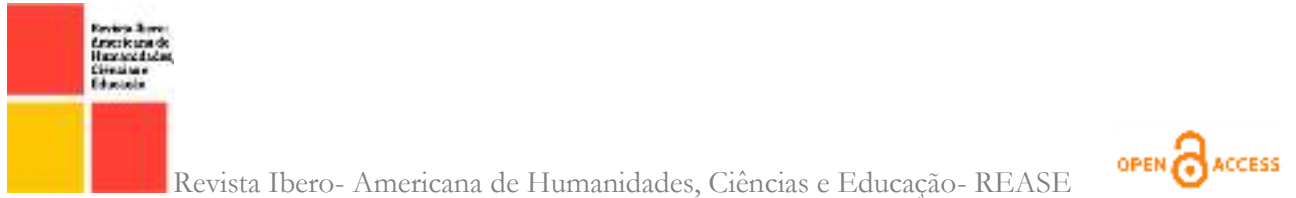

direcionados à definição de conceitos, identificação de lacunas nas áreas de estudos, revisão de teorias e análise metodológica dos estudos sobre um determinado tópico, tem como finalidade sintetizar resultados obtidos em pesquisas sobre um tema ou questão, de maneira sistemática, ordenada e abrangente (CASARIN et al, 2020).

Este estudo foi formado com base em uma revisão da literatura realizada no período entre fevereiro e outubro de 2021, utilizou-se o método qualitativo e pesquisa bibliográfica, no qual foram adotados materiais devidamente publicados (artigos e trabalho de conclusão de curso) disponíveis em baeses de dados como Scielo (Scientific Electronic Library Online) e Google Acadêmico. Os descritores utilizados na pesquisa foram: assistência farmacêutica, atenção farmacêutica, dispensação de medicamentos, drogarias, dispensação farmacêutica. Foi realizada a seleção dos artigos publicados entre 2004 e 2021 na língua portuguesa e que estavam disponíveis nas versões de acesso aberto, em um total de 3I artigos avaliados.

Os critérios de inclusão para seleção dos artigos foram realizados de acordo com o assunto proposto priorizando as publicações submetidas atualmente, ou seja, aquelas que tinham sido publicadas recentemente e que tinham abordagem clara sobre o tema. Já os de exclusão foram relacionados, aos artigos com acesso limitado ou que não estavam bem definidos com o tema, ou seja, não se enquadravam nos critérios de inclusão citados. Utilizou-se documentos com um maior período por considerar relevante todo o contexto histórico da profissão na abordagem do tema dispensação farmacêuticos.

\section{RESULTADOS E DISCUSSÃO}

No último século houve um aumento significativo na expectativa de vida do ser humano e, como consequência, um considerável crescimento da população idosa. Entre os diversos motivos que contribuíram para este fato, está o desenvolvimento de novos e melhores medicamentos, assim como a ampliação do seu uso. No entanto, devido à diminuição das funções normais do 

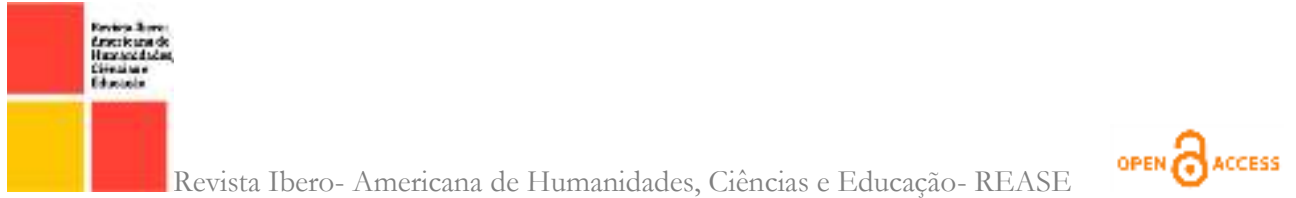

sistema imunológico, os idosos, por exemplo, tornam-se mais propensos ao desenvolvimento de doenças, devido às condições patológicas que normalmente acompanham o processo de envelhecimento, tais como uma maior incidência de doenças crônicas. Nessa perspectiva, a necessidade de maiores cuidados de saúde leva ao uso de um número cada vez maior de medicamentos (BATISTA et $\mathrm{al}, 2020$ ).

É de fundamental importância a criação de estratégias terapêuticas eficazes, com o intuito de interferir e racionalizar o uso de medicamentos. Estas práticas devem ser realizadas de forma constante para a população, com o objetivo de reduzir possíveis problemas relacionados ao uso inadequado de medicamentos, refletindo assim na melhoria da qualidade da saúde destas pessoas. A atuação do profissional farmacêutico é fundamental para tratar de todas as questões relativas ao uso de medicamentos, especialmente no contexto da polifarmácia (consumo simultâneo de múltiplos medicamentos) (BATISTA et $\mathrm{al}, 2020$ ).

Atenção Farmacêutica é um modelo de prática farmacêutica, desenvolvida no contexto da Assistência Farmacêutica. Compreende atitudes, valores éticos, comportamentos, habilidades, compromissos e corresponsabilidades na prevenção de doenças, promoção e recuperação da saúde, de forma integrada à equipe de saúde. É a interação direta do farmacêutico com o usuário, visando uma farmacoterapia racional que é quando o paciente faz o uso devido do medicamento para sua situação clínica dentro de suas necessidades individuais por um período estabelecido e a obtenção de resultados definidos e mensuráveis, voltados para a melhoria da qualidade de vida (BRASIL, 2002).

A Política Nacional de Medicamentos (PNM) tem como propósito garantir a necessária segurança, eficácia e qualidade do medicamento, além da promoção do uso racional e do acesso.

O papel chave do Farmacêutico é estender o caráter de beneficiário da Atenção Farmacêutica ao público, em seu conjunto, e reconhecer, deste modo, o 

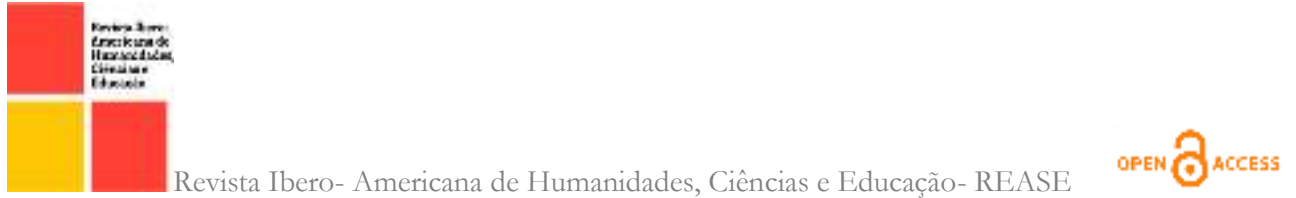

farmacêutico como dispensador da atenção sanitária que pode participar, ativamente, na prevenção das doenças e da promoção da saúde, junto com outros membros da equipe sanitária (OMS, 1994).

O farmacêutico perdeu seu espaço e reconhecimento nas farmácias comunitárias quando estas se tornaram simples estabelecimentos comerciais e de rede. Sua função como cuidador da saúde dos usuários de medicamentos não tem o mesmo valor, e a dispensação de medicamentos tornou-se um ato comercial. Em muitos estabelecimentos as atividades continuam centradas no medicamento, o que é evidenciado especialmente pela não adoção do novo modelo de prática, a Atenção Farmacêutica. O Conselho Federal de Farmácia (CFF) publica uma resolução, em 20or, que estabelece as Boas Práticas em Farmácia propondo outro conceito de assistência farmacêutica, que inclui a preocupação demonstrada nos informes da OMS sobre a assistência integral à saúde (ANGONESI, SEVALHO, 2oro). Que se relaciona com a definição de conceitos relacionados à prática farmacêutica na PNM.

Baseado na atuação profissional, na Atenção Farmacêutica, que se refere às atividades específicas do farmacêutico no âmbito da atenção à saúde. A atuação do profissional farmacêutico na orientação e prestação de serviços para apoiar usuários de medicamentos no uso correto e seguro desses produtos faz parte das vivências dos profissionais (ALMEIDA; MIRANDA, 2020; TORRES et al, 202I). Desde a sua proposição, muitas contribuições têm sido feitas no sentido de buscar o melhor entendimento e aplicação prática do conceito de Atenção Farmacêutica (ANGONESI, SEVALHO, 2oro).

Essa interação entre paciente e farmacêutico também deve envolver a concepção das necessidades biopsicossociais, sob a óptica da integralidade das ações de saúde, de cada indivíduo. E que também pode ser orientada a adesão ao tratamento não medicamentoso, pois este é um importante componente no tratamento de pacientes com doenças crônicas, por exemplo. De modo que é recomendado como forma de prevenção primária mudanças no estilo de vida (desenvolver rotinas saudáveis), tendo em vista que alterações deste tipo pode 

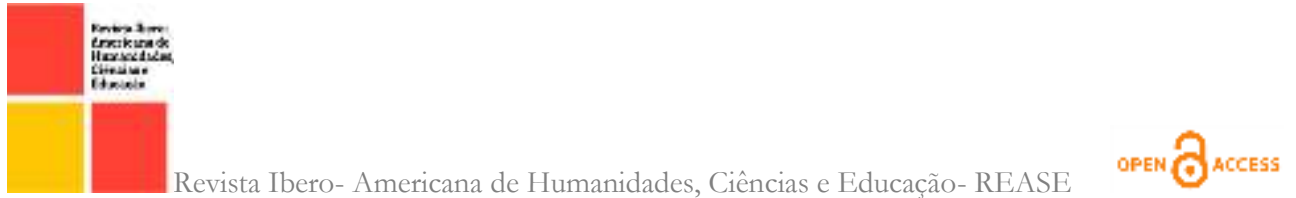

reduzir a mortalidade causada por alguma enfermidade cardiovascular. Algumas das recomendações englobam controlar o consumo de sódio, cigarro e bebidas alcoólicas; sempre que possível manter uma alimentação saudável rica em de potássio; sair do processo de sedentarismo, entre outros (ALMEIDA; MIRANDA, 2020; SANTOS et al, 2020).

Então, a Assistência Farmacêutica é o conjunto de ações voltadas para prevenção de doenças, tendo o medicamento como insumo essencial e visando o acesso ao seu uso racional às ações voltadas a promoção, proteção e recuperação da saúde, tanto individual como coletiva. Apresentando como alternativa farmacêutica o uso de medicamentos essenciais, envolvendo observação e avaliação de seu emprego, afim de que possa conceber resultados precisos que auxiliem na qualidade de vida da população (PEDRO et al, 2020).

O farmacêutico é responsável por satisfazer a necessidade que tem a sociedade de receber um tratamento apropriado, efetivo, seguro e cômodo, para isso é necessário que o profissional da Atenção Farmacêutica utilize em seu exercício um enfoque centrado no paciente, visto que, o cuidado ou assistência ao paciente no contexto da atenção farmacêutica requer uma relação que promova a participação do paciente no processo terapêutico. Nesta perspectiva, estabelece-se uma relação de reciprocidade de responsabilidades do profissional e do paciente baseada no diálogo, na confiança, respeito, sinceridade e autenticidade, com a finalidade específica de satisfazer as necessidades de uma assistência sanitária devidamente contextualizada na complexidade social (ANGONESI, SEVALHO, 2ого).

De acordo com Galato et al (2008), os pacientes que se dirigem a uma farmácia possuem a expectativa de encontrar um profissional farmacêutico bem vestido, de boa aparência, adequada formação acadêmica e com qualidades como inteligência, simpatia, honestidade, paciência e, que tenha conhecimentos e consistência no repasse de informações. Essas características do profissional apontadas por pacientes compreendem uma forma de estabelecerem a sua crença nos farmacêuticos dignos de confiança. E que esta confiança é obtida na 

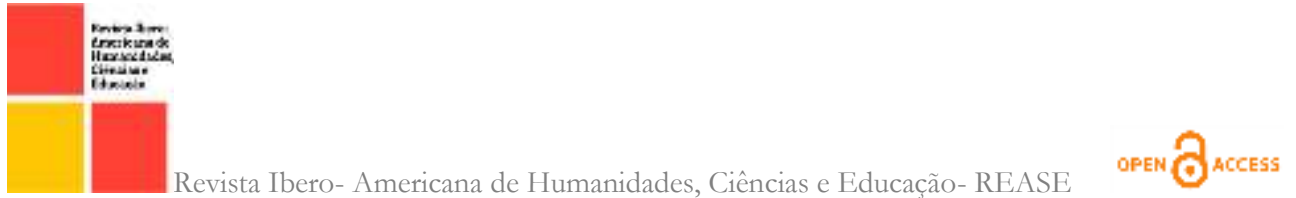

recepção ao paciente. Quando ele se aproxima do local de atendimento, o farmacêutico deve demonstrar consistentemente respeito, sensibilidade e dar prioridade ao mesmo, prestando atenção à sua comodidade física e emocional.

Apresentando também alguns fatores que podem interferir nesse momento de acolhimento, tais como: a falta de um espaço físico adequado e o pouco tempo disponível pelo profissional aos atendimentos dos pacientes devido à função gerencial da farmácia. Por isso, torna-se importante observar a área disponível ao atendimento, de forma a garantir um ambiente propício para estabelecer uma conversa com os pacientes. Ressaltando que pode ser importante dispor de um espaço totalmente privado para conversar com pacientes em situações especiais. Neste momento, o farmacêutico pode utilizarse de sua sensibilidade, adquirida por meio da prática profissional, para perceber a disponibilidade do paciente e despertar o interesse para o diálogo. De forma que, a dispensação do medicamento deve ser entendida como integrante do processo de atenção ao paciente (GALATO et al 2008).

A dispensação de medicamentos é o ato farmacêutico de distribuir um ou mais medicamentos a um paciente, geralmente como resposta à apresentação de uma prescrição elaborada por um profissional autorizado. Onde, é informado e orientado ao paciente o uso adequado do medicamento, como por exemplo, o cumprimento no regime das doses, a interação com alimentos ou outras medicações, condições de conservação do produto, reconhecimento de possíveis reações adversas, entre outros. De modo que, para se dispensar corretamente, é importante manter um diálogo entre o paciente e o profissional farmacêutico (ALMEIDA; MIRANDA, 2020).

Importante ressaltar sobre o que diz na Resolução de Diretoria Colegiada - RDC № 44, que estabelece os critérios e condições mínimas para o cumprimento das Boas Práticas Farmacêuticas para o controle sanitário do funcionamento, da dispensação e da comercialização de produtos e da prestação de serviços farmacêuticos em farmácias e drogarias. Dispõe também sobre a importância de um ambiente destinado aos serviços farmacêuticos deve ser 

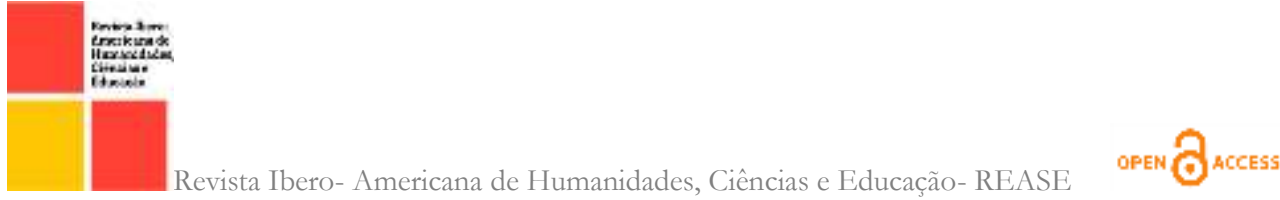

diverso daquele destinado à dispensação e à circulação de pessoas em geral, devendo o estabelecimento dispor de espaço específico para esse fim (BRASIL, 2009).

Segundo Galato et al (2008), na dispensação o farmacêutico distribuir um ou mais medicamentos a um paciente agindo de maneira ética respeitando a prescrição elaborada por um profissional autorizado, onde é entendida como integrante do processo de atenção ao paciente, ou seja, como uma atividade realizada por um profissional da saúde com foco na prevenção e promoção da saúde, tendo o medicamento como instrumento de ação. Sendo deste modo, uma oportunidade para o farmacêutico contribuir para o uso racional de medicamentos, visto que nesse momento de interação com o paciente é possível identificar a necessidade do mesmo e orientar tanto sobre o medicamento quanto sobre educação em saúde, atuando desta forma como um agente de saúde.

Dessa forma, o farmacêutico intervém e acompanha o tratamento farmacológico, tendo o usuário como foco de sua atuação profissional. Podendo desenvolver e incentivar a comunidade sobre condições que sejam determinantes para o seu estado de saúde. Quando a dispensação é acompanhada de orientação adequada, os riscos relativos a medicamentos diminuem, contribuindo para que os estabelecimentos farmacêuticos sejam, efetivamente, estabelecimentos de saúde (PEDRO et al, 2020).

Sendo importante observar que embora a legislação brasileira estabeleça que as farmácias tenham assistência de técnico responsável e que este deva permanecer durante todo o horário de funcionamento do estabelecimento, sabemos que na realidade encontramos vários estabelecimentos sem a devida responsabilidade técnica, seja pela falta de contratação ou ausência do profissional, de forma que, sem a supervisão do farmacêutico não pode haver dispensação de medicamentos, uma vez que a mesma é privativa deste profissional. Interferindo diretamente nas intervenções fundamentais para promover o uso racional de medicamentos, uma atividade profissional em que 

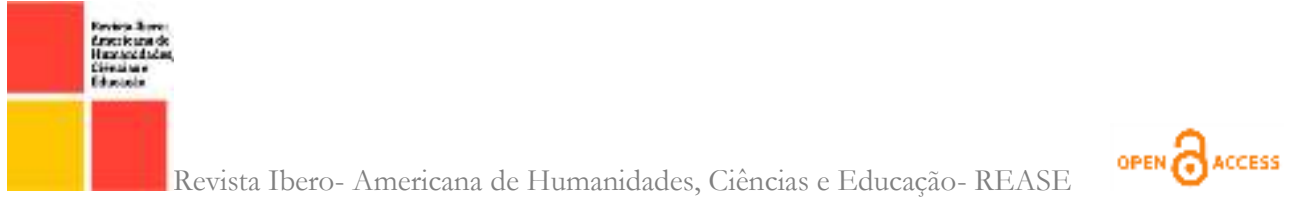

o paciente é o principal beneficiário das ações do farmacêutico. Pode-se analisar que as intervenções realizadas pelo profissional são capazes de desenvolver melhores resultados terapêuticos, garantindo segurança, eficácia e melhorias na farmacoterapia (PINTO et al, 2021; MILIOLI; ABREU, 202I).

A utilização de medicamentos pela população é um tema que merece destaque e faz parte das prioridades da OMS. No entanto, o controle da utilização adequada de medicamentos é uma questão antiga e de difícil operacionalização no Brasil, em razão de fatores de ordem econômica, cultural, de informação, educação e fiscalização (ALMEIDA; MIRANDA, 2020).

O farmacêutico tem como responsabilidade desenvolver seu papel perante a sociedade, proporcionando uma melhor qualidade de vida aos pacientes, trabalhando para que não haja problemas relacionados com medicamentos que poderiam ser evitados através de uma apresentação da farmacoterapia correta ao mesmo, o qual está apto para realizar esse acompanhamento. Ressaltando que além do conhecimento da atenção farmacêutica e farmácia clínica o profissional precisa ficar atentos com a qualidade de vida e satisfação dos pacientes (ALMEIDA; MIRANDA, 2020; SANTOS et al, 2020).

\section{CONCLUSÃO}

Um dos desafios do profissional farmacêutico é modificar as condutas, incorporando na prática um modelo que propicie ao farmacêutico assumir a responsabilidade com a farmacoterapia. Diante disso a relação contínua entre paciente e farmacêutico é fundamental para que os serviços de intervenção farmacêutica relacionados a terapia sejam realizados de maneira ética e legal, fornecendo resultados permanentes e assegurando a sua efetividade. Observouse que a prática da atenção farmacêutica em farmácias e drogarias requer modificação tanto técnica quanto administrativa, diante de tantos questionamentos levantados no presente estudo, ressalta-se a necessidade da transformação das farmácias em verdadeiros estabelecimentos de saúde. 


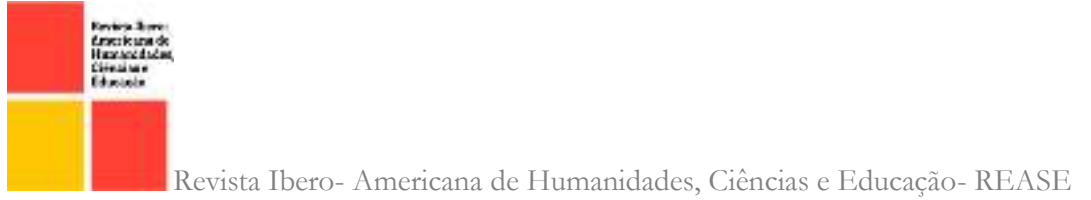

O reconhecimento da contribuição da atuação deste profissional para a obtenção de melhores resultados em saúde respalda a importância do farmacêutico nas drogarias e farmácias. Que ainda é visto como mero dispensador de medicamentos, voltado para a atividade comercial, visando os interesses financeiros da empresa a qual trabalha, deixando de lado seu papel que é promover, proteger e recuperar a saúde do paciente, através do uso racional de medicamentos. As farmácias e drogarias precisam ser vistas como locais de assistência à saúde da população e o farmacêutico deve resgatar seu papel frente a estes estabelecimentos.

Por meio deste estudo, foi possível evidenciar a importância da atividade do profissional farmacêutico, evitando assim a triste realidade de farmácias e drogarias serem vistas somente como locais de venda de medicamentos, onde não haveria a realização da assistência e atenção farmacêutica juntamente com o paciente, mas sim, apenas a entrega dos medicamentos. Em virtude dos aspectos mencionados, podemos dizer que através deste trabalho foi destacada a importância do farmacêutico no dia a dia das farmácias e drogarias, bem como no uso racional dos medicamentos.

\section{REFERÊNCIAS}

ALENCAR, T. O. S. et al. Dispensação farmacêutica: uma análise dos conceitos legais em relação à prática profissional. Revista de Ciências Farmacêuticas Básica e Aplicada, v. 32, n. I, 2011.

ALMEIDA, R. da C.; MIRANDA, C. V. de. A importância do farmacêutico na dispensação e controle de medicamentos classificados como antimicrobianos. Revista Saúde Multidisciplinar, v. 7, n. I, 2020.

ANGONESI, D.; SEVALHO, G.. Atenção Farmacêutica: fundamentação conceitual e crítica para um modelo brasileiro. Ciência \& saúde coletiva, v. I5, p. 3603-3614, 2010. 


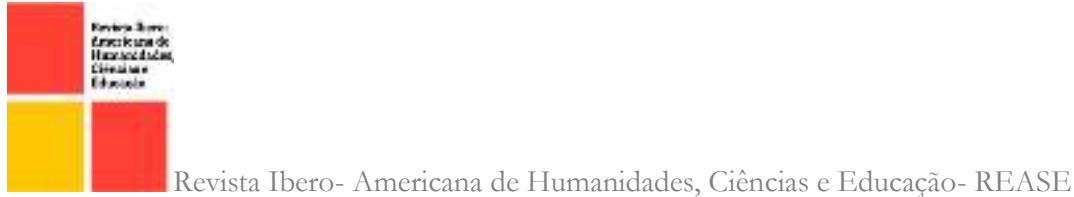

\section{open 8 access}

BATISTA, Sabrina de Cássia Macêdo et al. Polimedicação, atenção farmacêutica e cuidado farmacêutico. Journal of Biology \& Pharmacy and Agricultural Management, v. 16, n. 4, 2020.

BRASIL. Atenção farmacêutica no Brasil: trilhando caminhos: relatório 200I2002/ Adriana Mitsue Ivama [et al.]. - Brasília: Organização Pan-Americana da Saúde, 46 p., 2002.

BRASIL. Conselho Federal de Farmácia. Resolução no 357 de 20 de abril de 200 I (Alterada pela Resolução no 416/04). Aprova o regulamento técnico das Boas Práticas de Farmácia. Brasília, 200I.

BRASIL. Ministério da Saúde - MS. Agência Nacional de Vigilância Sanitária - ANVISA. - RDC № 44, de I7 de agosto de 2009. Dispõe sobre Boas Práticas Farmacêuticas. Brasília, 2009.

CASARIN, S. T. et al. Tipos de revisão de literatura: considerações das editoras do Journal of Nursing and Health/Types of literature review: considerations of the editors of the Journal of Nursing and Health. Journal of Nursing and Health, v. Io, n. 5, 2020.

GALATO, D. et al. A dispensação de medicamentos: uma reflexão sobre o processo para prevenção, identificação e resolução de problemas relacionados à farmacoterapia. Revista Brasileira de Ciências Farmacêuticas, v. 44, p. 465-475, 2008.

GASPAR, R. C. V.; MACHADO, V. F. L. S.. Automedicação X Prescrição Farmacêutica. Revista Científica Eletrônica de Ciências Aplicadas da FAIT. n. I. Maio, 2015.

MILIOLI, D. P. L. B.; ABREU, T. P. Atenção Farmacêutica na Drogaria. Revista Ibero-Americana de Humanidades, Ciências e Educação, v. 7, n. Io, p. 1069-1077, 2021.

OLIVEIRA, N. V. B. V. de et al. Atuação profissional dos farmacêuticos no Brasil: perfil sociodemográfico e dinâmica de trabalho em farmácias e drogarias privadas. Saúde e Sociedade, v. 26, p. IIO5-II2I, 2017. 
Organização Mundial da Saúde (OMS). O papel do farmacêutico no sistema de saúde. Genebra: Relatório de uma reunião da OMS. OMS, 24p. 1994. PEDRO, É. M. et al. A prática da atenção farmacêutica nas drogarias: revisão de literatura. Temas em Saúde. v. 20, N. 5, p. 48-64, João Pessoa, 2020. PINTO, G. R. S. et al. Contribuição farmacêutica na promoção da saúde em farmácias e drogarias. Research, Society and Development, v. Io, n. 3, p. e41910313614-e41910313614, 2021.

RODRIGUES, R. M. L. O papel do farmacêutico na assistência farmacêutica com ênfase na orientação quanto ao uso racional de medicamentos. 2019. 21 p. Trabalho de Conclusão de Curso (Título de especialista em Farmácia Hospitalar) - Centro Universitário CESMAC - Maceió - AL, 2019.

SANTOS, A. K. T. et al. Farmácia clínica e atenção farmacêutica em drogaria: Para hipertensos em uso de losartana potássica. Revista Eletrônica Gestão e Saúde, Vol. I. N. I. Ano I, 2020.

STEFANO, G. M. M. F; ARRUDA, R. C.. Dispensação de antimicrobianos: aspectos legais e autonomia farmacêutica. Revista Interdisciplinar em Gestão, Educação, Tecnologia e Saúde, v. I, n. ol, p. I14-126, 2018.

TORRES, P. C. et al. Experiências de usuários de um centro de informação de medicamentos sobre a atuação do profissional farmacêutico. Revista Brasileira de Farmácia Hospitalar e Serviços de Saúde, v. I2, n. 3, p. 617-617, 2021. ZUBIOLI, A. Ética farmacêutica. São Paulo: SOBRAVIME; 2004. 